Research Article

\title{
Some Refinements of Existence Results for SPDEs Driven by Wiener Processes and Poisson Random Measures
}

\begin{abstract}
Stefan Tappe
Institut für Mathematische Stochastik, Leibniz Universität Hannover, Welfengarten 1, 30167 Hannover, Germany

Correspondence should be addressed to Stefan Tappe, tappe@stochastik.uni-hannover.de

Received 4 June 2012; Accepted 6 August 2012

Academic Editor: Hari Mohan Srivástava

Copyright (C) 2012 Stefan Tappe. This is an open access article distributed under the Creative Commons Attribution License, which permits unrestricted use, distribution, and reproduction in any medium, provided the original work is properly cited.

We provide existence and uniqueness of global (and local) mild solutions for a general class of semilinear stochastic partial differential equations driven by Wiener processes and Poisson random measures under local Lipschitz and linear growth (or local boundedness, resp.) conditions. The socalled "method of the moving frame" allows us to reduce the SPDE problems to SDE problems.
\end{abstract}

\section{Introduction}

Semilinear stochastic partial differential equations (SPDEs) on Hilbert spaces, being of the type

$$
\begin{gathered}
Z_{t}=\left(A Z_{t}+\alpha\left(t, Z_{t}\right)\right) d t+\sigma\left(t, Z_{t}\right) d W_{t} \\
Z_{0}=z_{0},
\end{gathered}
$$

have widely been studied in the literature, see, for example, [1-4]. In (1.1), $A$ denotes the generator of a strongly continuous semigroup, and $W$ is a trace class Wiener process. In view of applications, this framework has been extended by adding jumps to the SPDE (1.1). More precisely, consider an SPDE of the type

$$
\begin{gathered}
d Z_{t}=\left(A Z_{t}+\alpha\left(t, Z_{t}\right)\right) d t+\sigma\left(t, Z_{t}\right) d W_{t}+\int_{E} \gamma\left(t, Z_{t-}, x\right)(\mu(d t, d x)-F(d x) d t), \\
Z_{0}=z_{0},
\end{gathered}
$$


where $\mu$ denotes a Poisson random measure on some mark space $(E, \varepsilon)$ with $d t \otimes F(d x)$ being its compensator. SPDEs of this type have been investigated in [5, 6], see also [7-12], where SPDEs with jump noises have been studied.

The goal of the present paper is to extend results and methods for SPDEs of the type (1.2) in the following directions.

(i) We consider more general SPDEs of the form

$$
\begin{aligned}
d Z_{t}= & \left(A Z_{t}+\alpha\left(t, Z_{t}\right)\right) d t+\sigma\left(t, Z_{t}\right) d W_{t}+\int_{B} \gamma\left(t, Z_{t-}, x\right)(\mu(d t, d x)-F(d x) d t) \\
& +\int_{B^{c}} \gamma\left(t, Z_{t-}, x\right) \mu(d t, d x) \\
Z_{0}= & z_{0}
\end{aligned}
$$

where $B \in \mathcal{E}$ is a set with $F\left(B^{c}\right)<\infty$. Then, the integral $\int_{B}$ represents the small jumps, and $\int_{B^{c}}$ represents the large jumps of the solution process. Similar SDEs have been considered in finite dimension in [13, Section II.2.c] and in infinite dimension in [14].

(ii) We will prove the following results (see Theorem 4.5) concerning existence and uniqueness of local and global mild solutions to (1.3):

(1) if $\left(\alpha, \sigma,\left.\gamma\right|_{B}\right)$ are locally Lipschitz and of linear growth, then existence and uniqueness of global mild solutions to (1.3) hold;

(2) if $\left(\alpha, \sigma,\left.\gamma\right|_{B}\right)$ are locally Lipschitz and locally bounded, then existence and uniqueness of local mild solutions to (1.3) hold;

(3) if $\left(\alpha, \sigma,\left.\gamma\right|_{B}\right)$ are locally Lipschitz, then uniqueness of mild solutions to (1.3) holds.

In particular, the result that local Lipschitz and linear growth conditions ensure existence and uniqueness of global mild solutions does not seem to be well known for SPDEs, as most of the mentioned references impose global Lipschitz conditions. An exception is [3], where the author treats Wiener process-driven SPDEs of the type (1.1), even on 2-smooth Banach spaces, and provides existence and uniqueness under local Lipschitz and linear growth conditions. In [3], the crucial assumption on the operator $A$ is that it generates an analytic semigroup, while our results hold true for every pseudocontractive semigroup.

(iii) We reduce the proofs of these SPDE results to the analysis of SDE problems. This is due to the "method of the moving frame", which has been presented in [6]. As a direct consequence, we obtain that any mild solution to (1.3) is càdlàg. 
As just mentioned, we will utilize the "method of the moving frame" from [6], which allows us to reduce the SPDE problems to SDE problems. Therefore, we will be concerned with SDEs in Hilbert spaces being of the type

$$
\begin{gathered}
d Y_{t}=a\left(t, Y_{t}\right) d t+b\left(t, Y_{t}\right) d W_{t}+\int_{B} c\left(t, Y_{t-}, x\right)(\mu(d t, d x)-F(d x) d t)+\int_{B^{c}} c\left(t, Y_{t-}, x\right) \mu(d t, d x), \\
Y_{0}=y_{0} .
\end{gathered}
$$

By using the technique of interlacing solutions at jump times (which, in particular cases has been applied, e.g., in [15, Section 6.2] and [10, Section 9.7]), we can reduce the SDE (1.4) to SDEs of the form

$$
\begin{gathered}
d Y_{t}=a\left(t, Y_{t}\right) d t+b\left(t, Y_{t}\right) d W_{t}+\int_{B} c\left(t, Y_{t-}, x\right)(\mu(d t, d x)-F(d x) d t), \\
Y_{0}=y_{0},
\end{gathered}
$$

without large jumps, and for those SDEs, suitable techniques and results are available in the literature. This allows us to derive existence and uniqueness results for the SDE (1.4), which are subject to the regularity conditions described above. We point out that [14] also studies Hilbert space-valued SDEs of the type (1.4) and provides an existence and uniqueness result considerably going beyond the classical results which impose global Lipschitz conditions. In Section 3.3, we provide a comparison of our existence and uniqueness result for SDEs of the type (1.4) with that from [14].

The remainder of this paper is organized as follows: in Section 2, we provide the required preliminaries and notation. In Section 3, we prove existence and uniqueness results for (local) strong solutions to SDEs of the form (1.4), and in Section 4, we prove existence and uniqueness results for (local) mild solutions to SPDEs of the form (1.3) by using the "method of the moving frame."

\section{Preliminaries and Notation}

In this section, we provide the required preliminary results and some basic notation.

Throughout this text, let $(\Omega, \mathscr{F}, \mathbb{F}, \mathbb{P})$ with $\mathbb{F}=\left(\mathscr{F}_{t}\right)_{t \geq 0}$ be a filtered probability space satisfying the usual conditions.

Let $U$ be a separable Hilbert space, and let $Q \in L(U)$ be a nuclear, self-adjoint, positive definite linear operator. Then, there exist an orthonormal basis $\left(e_{j}\right)_{j \in \mathbb{N}}$ of $U$ and a sequence $\left(\lambda_{j}\right)_{j \in \mathbb{N}} \subset(0, \infty)$ with $\sum_{j \in \mathbb{N}} \lambda_{j}<\infty$ such that

$$
Q e_{j}=\lambda_{j} e_{j} \quad \forall j \in \mathbb{N},
$$


namely, the $\lambda_{j}$ are the eigenvalues of $Q$, and each $e_{j}$ is an eigenvector corresponding to $\lambda_{j}$. The space $U_{0}:=Q^{1 / 2}(U)$, equipped with the inner product

$$
\langle u, v\rangle_{U_{0}}:=\left\langle Q^{-1 / 2} u, Q^{-1 / 2} v\right\rangle_{U^{\prime}}
$$

is another separable Hilbert space, and $\left(\sqrt{\lambda_{j}} e_{j}\right)_{j \in \mathbb{N}}$ is an orthonormal basis. Let $W$ be a $U$ valued $Q$-Wiener process, see [1, page 86, 87]. For another separable Hilbert space $H$, we denote by $L_{2}^{0}(H):=L_{2}\left(U_{0}, H\right)$ the space of Hilbert-Schmidt operators from $U_{0}$ into $H$, which, endowed with the Hilbert-Schmidt norm

$$
\|\Phi\|_{L_{2}^{0}(H)}:=\left(\sum_{j \in \mathbb{N}}\left\|\Phi\left(\sqrt{\lambda_{j}} e_{j}\right)\right\|^{2}\right)^{1 / 2}, \quad \Phi \in L_{2}^{0}(H),
$$

itself is a separable Hilbert space.

Let $(E, \varepsilon)$ be a measurable space which we assume to be a Blackwell space (see [16, 17]). We remark that every Polish space with its Borel $\sigma$-field is a Blackwell space. Furthermore, let $\mu$ be a time-homogeneous Poisson random measure on $\mathbb{R}_{+} \times E$, see [13, Definition II.1.20]. Then its compensator is of the form $d t \otimes F(d x)$, where $F$ is a $\sigma$-finite measure on $(E, \varepsilon)$.

For the following definitions, let $\tau$ be a finite stopping time.

(i) We define the new filtration $\mathbb{F}^{(\tau)}=\left(\mathcal{F}_{t}^{(\tau)}\right)_{t \geq 0}$ by

$$
\mathcal{F}_{t}^{(\tau)}:=\mathcal{F}_{\tau+t}, \quad t \geq 0
$$

(ii) We define the new $U$-valued process $W^{(\tau)}$ by

$$
W_{t}^{(\tau)}:=W_{\tau+t}-W_{\tau}, \quad t \geq 0 .
$$

(iii) We define the new random measure $\mu^{(\tau)}$ on $\mathbb{R}_{+} \times E$ by

$$
\mu^{(\tau)}(\omega ; B):=\mu\left(\omega ; B_{\tau(\omega)}\right), \quad \omega \in \Omega, B \in \mathbb{B}\left(\mathbb{R}_{+}\right) \otimes \mathcal{\varepsilon},
$$

where we use the notation

$$
B_{\tau}:=\left\{(t+\tau, x) \in \mathbb{R}_{+} \times E:(t, x) \in B\right\} .
$$

Then, $W^{(\tau)}$ is an $\mathbb{F}^{(\tau)}$-adapted $Q$-Wiener process, and $\mu^{(\tau)}$ is a time-homogeneous Poisson random measure relative to the filtration $\mathbb{F}^{(\tau)}$ with compensator $d t \otimes F(d x)$, cf. [18, Lemma 4.6].

Lemma 2.1. Let $\varrho$ be another stopping time. Then, the mapping $(\rho-\tau)^{+}$is an $\mathbb{F}^{(\tau)}$-stopping time. 
Proof. For every $t \in \mathbb{R}_{+}$, we have

$$
\left\{(\rho-\tau)^{+} \leq t\right\}=\{\rho-\tau \leq t\}=\{\rho \leq \tau+t\} \in \mathcal{F}_{\tau+t}=\mathscr{F}_{t}^{(\tau)},
$$

showing that $(\rho-\tau)^{+}$is an $\mathbb{F}^{(\tau)}$-stopping time.

Denoting by $p^{(\tau)}$ the predictable $\sigma$-algebra relative to the filtration $\mathbb{F}^{(\tau)}$, we have the following auxiliary result.

Lemma 2.2. The following statements are true:

(1) the mapping

$$
\theta_{\tau}: \Omega \times \mathbb{R}_{+} \longrightarrow \Omega \times \mathbb{R}_{+}, \quad \theta_{\tau}(\omega, t):=(\omega, \tau(\omega)+t)
$$

is $p^{(\tau)}-p$-measurable.

(2) the mapping

$$
\vartheta_{\tau}: \Omega \longrightarrow \Omega \times \mathbb{R}_{+}, \quad \vartheta_{\tau}(\omega):=(\omega, \tau(\omega))
$$

is $\mathcal{F}_{\tau}$-D-measurable.

Proof. According to [13, Theorem I.2.2], the system of sets

$$
\left\{A \times\{0\}: A \in \mathcal{F}_{0}\right\} \cup\{\llbracket 0, \varrho \rrbracket: \varrho \text { is a stopping time }\}
$$

is a generating system of the predictable $\sigma$-algebra $\not$. For any set $A \in \mathcal{F}_{0}$, we have

$$
\theta_{\tau}^{-1}(A \times\{0\})=(A \cap\{\tau=0\}) \times\{0\} \in D^{(\tau)} .
$$

Furthermore, for any $\mathbb{F}$-stopping time $\varrho$, we have

$$
\begin{aligned}
\theta_{\tau}^{-1}(\llbracket 0, \varrho \rrbracket) & =\theta_{\tau}^{-1}\left(\left\{(\omega, t) \in \Omega \times \mathbb{R}_{+}: 0 \leq t \leq \rho(\omega)\right\}\right) \\
& =\left\{(\omega, t) \in \Omega \times \mathbb{R}_{+}: 0 \leq \tau(\omega)+t \leq \rho(\omega)\right\} \\
& =\left\{(\omega, t) \in \Omega \times \mathbb{R}_{+}: 0 \leq t \leq \rho(\omega)-\tau(\omega)\right\} \\
& =\llbracket 0, \rho-\tau \rrbracket=\llbracket 0,(\rho-\tau)^{+} \rrbracket \backslash(\{\tau>\rho\} \times\{0\}) \in D^{(\tau)},
\end{aligned}
$$

where, in the last step, we have used Lemma 2.1. This proves the first statement.

According to [13, Theorem I.2.2], the system of sets

$$
\left\{A \times\{0\}: A \in \mathcal{F}_{0}\right\} \cup\left\{A \times(s, t]: s<t, A \in \mathcal{F}_{s}\right\}
$$


is a generating system of the predictable $\sigma$-algebra $D$. For any set $A \in \mathcal{F}_{0}$, we have

$$
\vartheta_{\tau}^{-1}(A \times\{0\})=A \cap\{\tau=0\} \in \mathcal{F}_{0} \subset \mathcal{F}_{\tau} .
$$

Furthermore, for all $s, t \in \mathbb{R}_{+}$with $s<t$ and $A \in \mathcal{F}_{s}$, we have

$$
\vartheta_{\tau}^{-1}(A \times(s, t])=A \cap\{s<\tau\} \cap\{\tau \leq t\} \in \mathcal{F}_{\tau}
$$

establishing the second statement.

Let us further investigate the Poisson random measure $\mu$. According to [13, Proposition II.1.14], there exist a sequence $\left(\kappa_{n}\right)_{n \in \mathbb{N}}$ of finite stopping times with $\llbracket \kappa_{n} \rrbracket \cap \llbracket \kappa_{m} \rrbracket=\emptyset$ for $n \neq m$ and an $E$-valued optional process $\xi$ such that for every optional process $\gamma: \Omega \times \mathbb{R}_{+} \times E \rightarrow$ $H$, where $H$ denotes a separable Hilbert space, and all $0 \leq t \leq u$ with

$$
\mathbb{P}\left(\int_{t}^{u} \int_{E}\|\gamma(s, x)\| \mu(d s, d x)<\infty\right)=1
$$

we have

$$
\int_{t}^{u} \int_{E} \gamma(s, x) \mu(d s, d x)=\sum_{n \in \mathbb{N}} \gamma\left(\kappa_{n}, \xi_{\kappa_{n}}\right) \mathbb{1}_{\left\{t<\kappa_{n} \leq u\right\}} .
$$

Let $B \in \mathcal{\varepsilon}$ be a set with $F\left(B^{c}\right)<\infty$. We define the mappings $\varrho_{k}: \Omega \rightarrow \overline{\mathbb{R}}_{+}, k \in \mathbb{N}_{0}$ as

$$
\rho_{k}:=\inf \left\{t \geq 0: \mu\left([0, t] \times B^{c}\right)=k\right\}, \quad k \in \mathbb{N}_{0} .
$$

Lemma 2.3. The following statements are true:

(1) for each $k \in \mathbb{N}$, the mapping $Q_{k}$ is a finite stopping time,

(2) one has $\varphi_{0}=0$ and $\mathbb{P}\left(\rho_{k}<\rho_{k+1}\right)=1$ for all $k \in \mathbb{N}_{0}$,

(3) one has $\mathbb{P}\left(\rho_{k} \rightarrow \infty\right)=1$.

Proof. This follows from [19, Lemma A.19].

\section{Existence and Uniqueness of Strong Solutions to Hilbert Space-Valued SDEs}

In this section, we establish existence and uniqueness of (local) strong solutions to Hilbert space-valued SDEs of the type (1.4).

Let $\mathscr{t}$ be a separable Hilbert space, and let $B \in \mathcal{E}$ be a set with $F\left(B^{c}\right)<\infty$. Furthermore, let $a: \Omega \times \mathbb{R}_{+} \times \mathscr{H} \rightarrow \mathscr{H}$ and $b: \Omega \times \mathbb{R}_{+} \times \mathscr{H} \rightarrow L_{2}^{0}(\mathscr{H})$ be $D \otimes B(\mathscr{H})$-measurable mappings, and let $c: \Omega \times \mathbb{R}_{+} \times \mathscr{H} \times E \rightarrow \mathscr{H}$ be a $D \otimes B(\mathscr{H}) \otimes \mathcal{E}$-measurable mapping. 
Definition 3.1. One says that existence of (local) strong solutions to (1.4) holds, if for each $\mathcal{F}_{0^{-}}$ measurable random variable $y_{0}: \Omega \rightarrow \mathscr{H}$, there exists a (local) strong solution to (1.4) with initial condition $y_{0}$ (and some strictly positive lifetime $\tau>0$ ).

Definition 3.2. One says that uniqueness of (local) strong solutions to (1.4) holds, if for two (local) strong solutions to (1.4) with initial conditions $y_{0}$ and $y_{0}^{\prime}$ (and lifetimes $\tau$ and $\tau^{\prime}$ ) we have up to indistinguishability

$$
\begin{gathered}
\Upsilon_{\left\{y_{0}=y_{0}^{\prime}\right\}}=Y^{\prime} \mathbb{1}_{\left\{y_{0}=y_{0}^{\prime}\right\}}, \\
\left(Y^{\tau \wedge \tau^{\prime}} \mathbb{1}_{\left\{y_{0}=y_{0}^{\prime}\right\}}=\left(Y^{\prime}\right)^{\tau \wedge \tau^{\prime}} \mathbb{1}_{\left\{y_{0}=y_{0}^{\prime}\right\}}\right) .
\end{gathered}
$$

Note that uniqueness of local strong solutions to (1.4) implies uniqueness of strong solutions to (1.4). This is seen by setting $\tau:=\infty$ and $\tau^{\prime}:=\infty$.

Definition 3.3. One says that the mappings $\left(a, b,\left.c\right|_{B}\right)$ are locally Lipschitz if $\mathbb{P}$-almost surely

$$
\left(\int_{B}\|c(t, y, x)\|^{2} F(d x)\right)^{1 / 2}<\infty, \quad \forall t \in \mathbb{R}_{+} \text {and all } y \in \mathscr{H},
$$

and for each $n \in \mathbb{N}$, there is a nondecreasing function $L_{n}: \mathbb{R}_{+} \rightarrow \mathbb{R}_{+}$such that $\mathbb{P}$-almost surely

$$
\begin{gathered}
\left\|a\left(t, y_{1}\right)-a\left(t, y_{2}\right)\right\| \leq L_{n}(t)\left\|y_{1}-y_{2}\right\|, \\
\left\|b\left(t, y_{1}\right)-b\left(t, y_{2}\right)\right\|_{L_{2}^{0}(d)} \leq L_{n}(t)\left\|y_{1}-y_{2}\right\|, \\
\left(\int_{B}\left\|c\left(t, y_{1}, x\right)-c\left(t, y_{2}, x\right)\right\|^{2} F(d x)\right)^{1 / 2} \leq L_{n}(t)\left\|y_{1}-y_{2}\right\|,
\end{gathered}
$$

for all $t \in \mathbb{R}_{+}$and all $y_{1}, y_{2} \in \mathscr{H}$ with $\left\|y_{1}\right\|,\left\|y_{2}\right\| \leq n$.

Definition 3.4. One says that the mappings $\left(a, b,\left.c\right|_{B}\right)$ satisfy the linear growth condition if there exists a nondecreasing function $K: \mathbb{R}_{+} \rightarrow \mathbb{R}_{+}$such that $\mathbb{P}$-almost surely

$$
\begin{gathered}
\|a(t, y)\| \leq K(t)(1+\|y\|), \\
\|b(t, y)\|_{L_{2}^{0}(\mathscr{l})} \leq K(t)(1+\|y\|), \\
\left(\int_{B}\|c(t, y, x)\|^{2} F(d x)\right)^{1 / 2} \leq K(t)(1+\|y\|),
\end{gathered}
$$

for all $t \in \mathbb{R}_{+}$and all $y \in \mathscr{\ell}$. 
Definition 3.5. One says that the mappings $\left(a, b,\left.c\right|_{B}\right)$ are locally bounded if for each $n \in \mathbb{N}$, there is a nondecreasing function $M_{n}: \mathbb{R}_{+} \rightarrow \mathbb{R}_{+}$such that $\mathbb{P}$-almost surely

$$
\begin{gathered}
\|a(t, y)\| \leq M_{n}(t) \\
\|b(t, y)\|_{L_{2}^{0}(\mathfrak{d})} \leq M_{n}(t), \\
\left(\int_{B}\|c(t, y, x)\|^{2} F(d x)\right)^{1 / 2} \leq M_{n}(t),
\end{gathered}
$$

for all $t \in \mathbb{R}_{+}$and all $y \in \mathscr{H}$ with $\|y\| \leq n$.

For a finite stopping time $\tau$ and a set $\Gamma \in \mathcal{F}_{\tau}$, we define the mappings $a^{(\tau, \Gamma)}: \Omega \times \mathbb{R}_{+} \times$ $\mathscr{H} \rightarrow \mathscr{H}, b^{(\tau, \Gamma)}: \Omega \times \mathbb{R}_{+} \times \mathscr{H} \rightarrow L_{2}^{0}(\mathscr{H})$, and $c^{(\tau, \Gamma)}: \Omega \times \mathbb{R}_{+} \times \mathscr{H} \times E \rightarrow \mathscr{H}$ as

$$
\begin{gathered}
a^{(\tau, \Gamma)}(t, y):=a(\tau+t, y) \mathbb{1}_{\Gamma}, \\
b^{(\tau, \Gamma)}(t, y):=b(\tau+t, y) \mathbb{1}_{\Gamma}, \\
c^{(\tau, \Gamma)}(t, y, x):=c(\tau+t, y, x) \mathbb{1}_{\Gamma} .
\end{gathered}
$$

By Lemma 2.2, the mappings $a^{(\tau, \Gamma)}$ and $b^{(\tau, \Gamma)}$ are $p^{(\tau)} \otimes B(\mathscr{L})$-measurable, and $c^{(\tau, \Gamma)}$ is $p^{(\tau)} \otimes$ $B(\mathscr{L}) \otimes \mathcal{E}$-measurable. We will also use the notation

$$
a^{(\tau)}:=a^{(\tau, \Omega)}, \quad b^{(\tau)}:=b^{(\tau, \Omega)}, \quad c^{(\tau)}:=c^{(\tau, \Omega)} .
$$

Lemma 3.6. Suppose that $\tau \mathbb{1}_{\Gamma}$ is bounded. Then, the following statements are true:

(1) if $\left(a, b,\left.c\right|_{B}\right)$ are locally Lipschitz, then $\left(a^{(\tau, \Gamma)}, b^{(\tau, \Gamma)},\left.c^{(\tau, \Gamma)}\right|_{B}\right)$ are locally Lipschitz, too;

(2) if $\left(a, b,\left.c\right|_{B}\right)$ satisfy the linear growth condition, then $\left(a^{(\tau, \Gamma)}, b^{(\tau, \Gamma)},\left.c^{(\tau, \Gamma)}\right|_{B}\right)$ satisfy the linear growth condition, too.

Proof. Suppose that $\left(a, b,\left.c\right|_{B}\right)$ satisfy the linear growth condition. Since $\tau \mathbb{1}_{\Gamma}$ is bounded, there exists a constant $T \geq 0$ such that $\tau \mathbb{1}_{\Gamma} \leq T$. The mapping $\tilde{K}:=K(\bullet+T): \mathbb{R}_{+} \rightarrow \mathbb{R}_{+}$is nondecreasing, and we have $\mathbb{P}$-almost surely

$$
\left\|a^{(\tau, \Gamma)}(t, y)\right\|=\left\|a(t+\tau, y) \mathbb{1}_{\Gamma}\right\| \leq K(t+\tau) \mathbb{1}_{\Gamma}(1+\|y\|) \leq \tilde{K}(t)(1+\|y\|),
$$

for all $t \in \mathbb{R}_{+}$and $y \in \mathscr{H}$. Analogous estimates for $b^{(\tau, \Gamma)}$ and $c^{(\tau, \Gamma)}$ prove that $\left(a^{(\tau, \Gamma)}\right.$, $\left.b^{(\tau, \Gamma)},\left.c^{(\tau, \Gamma)}\right|_{B}\right)$ satisfy the linear growth condition, too. The remaining statement is proven analogously. 
International Journal of Stochastic Analysis

Lemma 3.7. Let $\tau$ and $\rho$ be two finite stopping times, and let $\Gamma \in \mathcal{F}_{\tau}$ be a set with $\Gamma \subset\{\tau \leq \rho\}$. If $\Upsilon$ is an $\mathbb{F}$-adapted local strong solution to (1.4) with lifetime $Q$, then

$$
Y^{(\tau, \Gamma)}:=Y_{\tau+\bullet} \mathbb{1}_{\Gamma}
$$

is an $\mathbb{F}^{(\tau)}$-adapted local strong solution to (1.4) with parameters

$$
a=a^{(\tau, \Gamma)}, \quad b=b^{(\tau, \Gamma)}, \quad c=c^{(\tau, \Gamma)}, \quad W=W^{(\tau)}, \quad \mu=\mu^{(\tau)},
$$

initial condition $Y_{\tau} \mathbb{1}_{\Gamma}$, and lifetime $(\rho-\tau)^{+}$.

Proof. The process $Y^{(\tau, \Gamma)}$ given by (3.11) is $\mathbb{F}^{(\tau)}$ adapted, and we have

$$
\begin{aligned}
Y_{t}^{(\tau, \Gamma)} \mathbb{1}_{\llbracket 0,(\rho-\tau)^{\dagger} \rrbracket}(t)= & Y_{\tau++} \mathbb{1}_{\Gamma} \mathbb{1}_{\llbracket 0,(\rho-\tau)^{\dagger} \rrbracket}(t)=\left[Y_{\tau}+\left(Y_{\tau+t}-Y_{\tau}\right)\right] \mathbb{1}_{\Gamma} \mathbb{1}_{\llbracket 0,(\rho-\tau)^{+} \rrbracket}(t) \\
= & {\left[Y_{\tau}+\int_{\tau}^{\tau+t} a\left(s, Y_{s}\right) d s+\int_{\tau}^{\tau+t} b\left(s, Y_{s}\right) d W_{s}\right.} \\
& +\int_{\tau}^{\tau+t} \int_{B} c\left(s, Y_{s-} x\right)(\mu(d s, d x)-F(d x) d s) \\
& \left.+\int_{\tau}^{\tau+t} \int_{B^{c}} c\left(s, Y_{s-} x\right) \mu(d s, d x)\right] \mathbb{1}_{\Gamma} \mathbb{1}_{\llbracket 0,(\rho-\tau)^{+} \rrbracket}(t) .
\end{aligned}
$$

Therefore, we obtain

$$
\begin{aligned}
Y_{t}^{(\tau, \Gamma)} \mathbb{1}_{\llbracket 0,(\varrho-\tau)^{+} \rrbracket}(t)=\left[Y_{\tau} \mathbb{1}_{\Gamma}+\int_{0}^{t} a\left(\tau+s, Y_{\tau+s}\right) \mathbb{1}_{\Gamma} d s+\int_{0}^{t} b\left(\tau+s, Y_{\tau+s}\right) \mathbb{1}_{\Gamma} d W_{s}^{(\tau)}\right. \\
+\int_{0}^{t} \int_{B} c\left(\tau+s, Y_{(\tau+s)-}, x\right) \mathbb{1}_{\Gamma}\left(\mu^{(\tau)}(d s, d x)-F(d x) d s\right) \\
\left.+\int_{0}^{t} \int_{B^{c}} c\left(\tau+s, Y_{(\tau+s)-}, x\right) \mathbb{1}_{\Gamma} \mu^{(\tau)}(d s, d x)\right] \mathbb{1}_{\llbracket 0,(\rho-\tau)^{+} \rrbracket}(t) .
\end{aligned}
$$

Taking into account the Definitions (3.6)-(3.8) of $a^{(\tau, \Gamma)}, b^{(\tau, \Gamma)}, c^{(\tau, \Gamma)}$ and the Definition (3.11) of $Y^{(\tau, \Gamma)}$, it follows that

$$
\begin{aligned}
Y_{t}^{(\tau, \Gamma)} \mathbb{1}_{\llbracket 0,(\rho-\tau)^{\dagger} \rrbracket}(t)=\left[Y_{\tau} \mathbb{1}_{\Gamma}+\int_{0}^{t} a^{(\tau, \Gamma)}\left(s, Y_{s}^{(\tau, \Gamma)}\right) d s+\int_{0}^{t} b^{(\tau, \Gamma)}\left(s, Y_{s}^{(\tau, \Gamma)}\right) d W_{s}^{(\tau)}\right. \\
\quad+\int_{0}^{t} \int_{B} c^{(\tau)}\left(s, Y_{s-}^{(\tau, \Gamma)}, x\right)\left(\mu^{(\tau)}(d s, d x)-F(d x) d s\right) \\
\left.+\int_{0}^{t} \int_{B^{c}} c^{(\tau)}\left(s, Y_{s-}^{(\tau, \Gamma)}, x\right) \mu^{(\tau)}(d s, d x)\right] \mathbb{1}_{\llbracket 0,(\rho-\tau)^{+} \rrbracket}(t) .
\end{aligned}
$$


Consequently, $Y^{(\tau, \Gamma)}$ is a local strong solution to (1.4) with parameters (3.12), initial condition $Y_{\tau} \mathbb{1}_{\Gamma}$, and lifetime $(\rho-\tau)^{+}$.

Lemma 3.8. Let $\tau \leq \rho$ be two finite stopping times. If $Y^{(0)}$ is an $\mathbb{F}$-adapted local strong solution to (1.4) with lifetime $\tau$, and $Y^{(\tau)}$ is an $\mathbb{F}^{(\tau)}$-adapted local strong solution to (1.4) with parameters

$$
a=a^{(\tau)}, \quad b=b^{(\tau)}, \quad c=c^{(\tau)}, \quad W=W^{(\tau)}, \quad \mu=\mu^{(\tau)},
$$

initial condition $Y_{\tau}^{(0)}$, and lifetime $\rho-\tau$, then

$$
Y:=Y^{(0)} \mathbb{1}_{\llbracket 0, \tau \rrbracket}+Y_{\bullet-\tau}^{(\tau)} \mathbb{1} \rrbracket \tau, \wp \rrbracket
$$

is an $\mathbb{F}$-adapted local strong solution to (1.4) with lifetime $\rho$.

Proof. Let $t \in \mathbb{R}_{+}$be arbitrary. Then, the random variable $Y_{t}^{(0)} \mathbb{1}_{\{\tau \geq t\}}$ is $\mathcal{F}_{t}$-measurable. Let $C \in$ $B(\mathcal{H})$ be an arbitrary Borel set. We define $D_{C} \in \mathcal{F}_{t}$ as

$$
D_{C}:= \begin{cases}(\{\tau<t\} \cap\{t \leq \rho\})^{c} & \text { if } 0 \in C, \\ \emptyset & \text { if } 0 \notin C .\end{cases}
$$

According to Lemma 2.1, the mapping $(t-\tau)^{+}$is an $\mathbb{F}^{(\tau)}$-stopping time. Therefore, we get

$$
\left\{Y_{(t-\tau)^{+}}^{(\tau)} \in C\right\} \in \mathcal{F}_{(t-\tau)^{+}}^{(\tau)}=\boldsymbol{F}_{\tau+(t-\tau)^{+}},
$$

and hence, we obtain

$$
\begin{aligned}
\left\{Y_{t-\tau}^{(\tau)} \mathbb{1}_{\{\tau<t \leq \rho\}} \in C\right\} & =\left\{Y_{(t-\tau)^{+}}^{(\tau)} \mathbb{1}_{\{\tau<t \leq \rho\}} \in C\right\} \\
& =\left(\{\tau<t\} \cap\{t \leq \rho\} \cap\left\{Y_{(t-\tau)^{+}}^{(\tau)} \in C\right\}\right) \cup D_{C} \\
& =\left(\{\rho \geq t\} \cap\{\tau \neq t\} \cap\{\tau \leq t\} \cap\left\{Y_{(t-\tau)^{+}}^{(\tau)} \in C\right\}\right) \cup D_{C} \\
& =\left(\{\rho \geq t\} \cap\{\tau \neq t\} \cap\left\{\tau+(t-\tau)^{+}=t\right\} \cap\left\{Y_{(t-\tau)^{+}}^{(\tau)} \in C\right\}\right) \cup D_{C} \in \mathcal{F}_{t},
\end{aligned}
$$


showing that the process $Y$ defined in (3.17) is $\mathbb{F}$ adapted. Moreover, since $Y^{(\tau)}$ is local strong solution to (1.4) with initial condition $Y_{\tau}^{(0)}$ and lifetime $\rho-\tau$, we have

$$
\begin{aligned}
Y_{t-\tau}^{(\tau)} \mathbb{1}_{\rrbracket \tau, \rho \rrbracket}(t)=\left[Y_{\tau}^{(0)}\right. & +\int_{0}^{t-\tau} a^{(\tau)}\left(s, Y_{s}^{(\tau)}\right) d s+\int_{0}^{t-\tau} b^{(\tau)}\left(s, Y_{s}^{(\tau)}\right) d W_{s}^{(\tau)} \\
& +\int_{0}^{t-\tau} \int_{B} c^{(\tau)}\left(s, Y_{s-}^{(\tau)}, x\right)\left(\mu^{(\tau)}(d s, d x)-F(d x) d s\right) \\
& \left.+\int_{0}^{t-\tau} \int_{B^{c}} c^{(\tau)}\left(s, Y_{s^{-}}^{(\tau)}, x\right) \mu^{(\tau)}(d s, d x)\right] \mathbb{1}_{\rrbracket \tau, \rho \rrbracket}(t) .
\end{aligned}
$$

By the Definitions (3.6)-(3.9) of $a^{(\tau)}, b^{(\tau)}, c^{(\tau)}$, we obtain

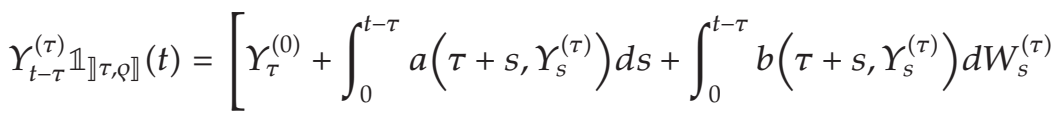

$$
\begin{aligned}
& +\int_{0}^{t-\tau} \int_{B} c\left(\tau+s, Y_{s-}^{(\tau)}, x\right)\left(\mu^{(\tau)}(d s, d x)-F(d x) d s\right) \\
& \left.+\int_{0}^{t-\tau} \int_{B^{c}} c\left(\tau+s, Y_{s-}^{(\tau)}, x\right) \mu^{(\tau)}(d s, d x)\right] \mathbb{1}_{\rrbracket \tau, \rho \rrbracket}(t) .
\end{aligned}
$$

Therefore, we get

$$
\begin{aligned}
& Y_{t-\tau}^{(\tau)} \mathbb{1}_{\rrbracket \tau, \rho \rrbracket}(t)=\left[Y_{\tau}^{(0)}+\int_{\tau}^{t} a\left(s, Y_{s-\tau}^{(\tau)}\right) d s+\int_{\tau}^{t} b\left(s, Y_{s-\tau}^{(\tau)}\right) d W_{s}\right. \\
& +\int_{\tau}^{t} \int_{B} c\left(s, Y_{(s-\tau)-^{\prime}}^{(\tau)} x\right)(\mu(d s, d x)-F(d x) d s) \\
& \left.+\int_{\tau}^{t} \int_{B^{c}} c\left(s, Y_{(s-\tau)-^{\prime}}^{(\tau)} x\right) \mu(d s, d x)\right] \mathbb{1}_{\rrbracket \tau, \rho \rrbracket}(t) .
\end{aligned}
$$

By the Definition (3.17) of $Y$, we obtain

$$
\begin{aligned}
& Y_{t-\tau}^{(\tau)} \mathbb{1}_{\rrbracket \tau, \rho \rrbracket}(t)=\left[Y_{\tau}^{(0)}+\int_{\tau}^{t} a\left(s, Y_{s}\right) d s+\int_{\tau}^{t} b\left(s, Y_{s}\right) d W_{s}\right. \\
& +\int_{\tau}^{t} \int_{B} c\left(s, Y_{s^{-}}, x\right)(\mu(d s, d x)-F(d x) d s) \\
& \left.+\int_{\tau}^{t} \int_{B^{c}} c\left(s, Y_{s^{-}}, x\right) \mu(d s, d x)\right] \mathbb{1}_{\rrbracket \tau, \varrho \rrbracket}(t) .
\end{aligned}
$$


Since $Y^{(0)}$ is a local strong solution to (1.4) with lifetime $\tau$, we deduce that the process $Y$ given by (3.17) is a local strong solution to (1.4) with lifetime $\rho$.

Let $k \in \mathbb{N}_{0}$ be arbitrary. By Lemmas 2.1 and 2.3, the mapping $\varphi_{k+1}-\rho_{k}$ is a strictly positive $\mathbb{F}^{\left(\rho_{k}\right)}$-stopping time. Furthermore, let $\Gamma \in \mathcal{F}_{\rho_{k}}$ be arbitrary, and let $y_{0}^{\left(\rho_{k}\right)}: \Omega \rightarrow \mathscr{H}$ be an arbitrary $\mathcal{F}_{0}^{\left(\rho_{k}\right)}$-measurable random variable.

Lemma 3.9. If $Y^{\left(\rho_{k}, \Gamma\right)}$ is an $\mathbb{F}^{\left(\rho_{k}\right)}$-adapted local strong solution to (1.4) with parameters

$$
a=a^{\left(\varphi_{k}, \Gamma\right)}, \quad b=b^{\left(\rho_{k}, \Gamma\right)}, \quad c=c^{\left(\rho_{k}, \Gamma\right)}, \quad W=W^{\left(\rho_{k}\right)}, \quad \mu=\mu^{\left(\rho_{k}\right)},
$$

initial condition $y_{0}^{\left(\rho_{k}\right)} \mathbb{1}_{\Gamma}$, and lifetime $\tau$, then

$$
Y^{\left(\varrho_{k}, \Gamma\right)-}:=Y^{\left(\rho_{k}, \Gamma\right)}-c\left(\rho_{k+1}, Y_{\left(\rho_{k+1}-\rho_{k}\right){ }^{\prime}}^{\left(\rho_{k}, \Gamma\right)} \xi_{\rho_{k+1}}\right) \mathbb{1}_{\llbracket \rho_{k+1}-\rho_{k} \rrbracket} \mathbb{1}_{\left\{\rho_{k+1}-\varrho_{k} \leq \tau\right\}} \mathbb{1}_{\Gamma}
$$

is a $\mathbb{F}^{\left(\rho_{k}\right)}$-adapted local strong solution to (1.5) with parameters (3.25), initial condition $y_{0}^{\left(\rho_{k}\right)} \mathbb{1}_{\Gamma}$, and lifetime $\tau \wedge\left(\rho_{k+1}-\rho_{k}\right)$.

Proof. We define $J: \Omega \rightarrow \mathscr{H}$ as

$$
J:=c\left(\rho_{k+1}, Y_{\left(\rho_{k+1}-\rho_{k}\right)-^{\prime}}^{\left(\rho_{k}, \xi_{\rho_{k+1}}\right.}\right) \mathbb{1}_{\left\{\rho_{k+1}-\varrho_{k} \leq \tau\right\}} \mathbb{1}_{\Gamma}
$$

and the stochastic process $\left(J_{t}\right)_{t \geq 0}$ as $J_{t}:=J \mathbb{1}_{\llbracket \rho_{k+1}-\varrho_{k} \rrbracket}(t)$. By Lemma 2.2, the mapping $J$ is $\mathcal{F}_{\varphi_{k+1}}$ measurable. Let $C \in ß(\mathscr{\ell})$ be an arbitrary Borel set. We define $D_{C} \in \mathscr{f}_{t}$ as

$$
D_{C}:= \begin{cases}\left\{\rho_{k+1}-\rho_{k} \neq t\right\} & \text { if } 0 \in C, \\ \emptyset & \text { if } 0 \notin C .\end{cases}
$$

Then, for each $t \in \mathbb{R}_{+}$, we have

$$
\begin{aligned}
\left\{J_{t} \in C\right\} & =\left\{J \mathbb{1}_{\llbracket \rho_{k+1}-\rho_{k} \rrbracket}(t) \in C\right\}=\left(\{J \in C\} \cap\left\{\rho_{k+1}-\rho_{k}=t\right\}\right) \cup D_{C} \\
& =\left(\{J \in C\} \cap\left\{\rho_{k+1}=\varrho_{k}+t\right\}\right) \cup D_{C} \in \mathcal{F}_{\rho_{k}+t}=\mathcal{F}_{t}^{\left(\rho_{k}\right)} .
\end{aligned}
$$

Consequently, the process $Y^{\left(\rho_{k}, \Gamma\right)-}$ defined in (3.26) is $\mathbb{F}^{\left(\rho_{k}\right)}$-adapted. Furthermore, by the Definition (3.26), we have

$$
Y_{-}^{\left(\rho_{k}, \Gamma\right)} \mathbb{1}_{\llbracket 0, \tau \wedge\left(\rho_{k+1}-\rho_{k}\right) \rrbracket}=Y_{-}^{\left(\rho_{k}, \Gamma\right)-} \mathbb{1}_{\llbracket 0, \tau \wedge\left(\varphi_{k+1}-\rho_{k}\right) \rrbracket},
$$


and, by the Definition (3.8) of $c^{\left(\varphi_{k}, \Gamma\right)}$ and identity (2.18), we obtain

$$
\begin{aligned}
& \left(\int_{0}^{t} \int_{B^{c}} c^{\left(\rho_{k}, \Gamma\right)}\left(s, Y_{s-}^{\left(\rho_{k}, \Gamma\right)}, x\right) \mu^{\left(\rho_{k}\right)}(d s, d x)\right)_{\mathbb{1} \llbracket, \tau \wedge\left(\rho_{k+1}-\rho_{k}\right) \rrbracket}(t) \\
& =\left(\int_{0}^{t} \int_{B^{c}} c\left(\rho_{k}+s, Y_{s^{-}}^{\left(\rho_{k}, \Gamma\right)}, x\right) \mathbb{1}_{\Gamma} \mu^{\left(\rho_{k}\right)}(d s, d x)\right) \mathbb{1}_{\llbracket 0, \tau \wedge\left(\rho_{k+1}-\rho_{k}\right) \rrbracket}(t) \\
& =\left(\int_{\rho_{k}}^{\rho_{k}+t} \int_{B^{c}} c\left(s, Y_{\left(s-\rho_{k}\right)-^{\prime}}^{\left(\rho_{k}, \Gamma\right)}, x\right) \mathbb{1}_{\Gamma} \mu(d s, d x)\right)_{\llbracket\left[0, \tau \wedge\left(\rho_{k+1}-\rho_{k}\right) \rrbracket\right.}(t) \\
& =\left(\sum_{n \in \mathbb{N}} c\left(\kappa_{n}, Y_{\left.\left(\kappa_{n}-\rho_{k}\right){ }^{(}{ }^{(}, \Gamma\right)} \xi_{\kappa_{n}}\right) \mathbb{1}_{\left\{\xi_{\kappa_{n}} \notin B\right\}} \mathbb{1}_{\left\{\rho_{k}<\kappa_{n} \leq \varrho_{k}+t\right\}}\right) \mathbb{1}_{\llbracket 0, \tau \wedge\left(\rho_{k+1}-\varphi_{k}\right) \rrbracket}(t) \mathbb{1}_{\Gamma} \\
& =c\left(\rho_{k+1}, Y_{\left(\rho_{k+1}-\rho_{k}\right)-^{\prime}}^{\left(\rho_{k}, \xi_{\rho_{k+1}}\right.}\right) \mathbb{1}_{\llbracket \rho_{k+1}-\rho_{k} \rrbracket}(t) \mathbb{1}_{\left\{\rho_{k+1}-\rho_{k} \leq \tau\right\}} \mathbb{1}_{\Gamma},
\end{aligned}
$$

showing that $Y^{\left(\rho_{k}, \Gamma\right)-}$ is a local strong solution to (1.5) with parameters (3.25) and lifetime $\tau \wedge\left(\rho_{k+1}-\rho_{k}\right)$.

Lemma 3.10. If $Y^{\left(\rho_{k}, \Gamma\right)-}$ is an $\mathbb{F}^{\left(\rho_{k}\right)}$-adapted local strong solution to (1.5) with parameters (3.25), initial condition $y_{0}^{\left(\rho_{k}\right)} \mathbb{1}_{\Gamma}$, and lifetime $\tau$, then

$$
Y^{\left(\rho_{k}, \Gamma\right)}:=Y^{\left(\rho_{k}, \Gamma\right)-}+c\left(\rho_{k+1}, Y_{\left(\rho_{k+1}-\rho_{k}\right)-^{\prime}}^{\left(\rho_{k}, \Gamma\right)-} \xi_{\rho_{k+1}}\right) \mathbb{1}_{\llbracket \rho_{k+1}-\rho_{k} \rrbracket} \mathbb{1}_{\left\{\rho_{k+1}-\rho_{k} \leq \tau\right\}} \mathbb{1}_{\Gamma}
$$

is a $\mathbb{F}^{\left(Q_{k}\right)}$-adapted local strong solution to (1.4) with parameters (3.25), initial condition $y_{0}^{\left(\rho_{k}\right)} \mathbb{1}_{\Gamma}$, and lifetime $\tau \wedge\left(\rho_{k+1}-\rho_{k}\right)$.

Proof. The proof is analogous to that of Lemma 3.9.

\subsection{Uniqueness of Strong Solutions to Hilbert Space-Valued SDEs}

Now, we will deal with the uniqueness of strong solutions to the SDE (1.4).

Proposition 3.11. One supposes that the mappings $\left(a, b,\left.c\right|_{B}\right)$ are locally Lipschitz. Then, uniqueness of local strong solutions to (1.5) holds.

Proof. We can adopt a standard technique (see, e.g., the proof of Theorem 5.2.5 in [20]), where we apply the Itô isometry and Gronwall's lemma.

Theorem 3.12. One supposes that the mappings $\left(a, b,\left.c\right|_{B}\right)$ are locally Lipschitz. Then, uniqueness of local strong solutions to (1.4) holds. 
Proof. Let $Y$ and $Y^{\prime}$ be two local strong solutions to (1.5) with initial conditions $y_{0}$ and $y_{0}^{\prime}$, and lifetimes $\tau$ and $\tau^{\prime}$. By induction, we will prove that up to indistinguishability

$$
Y \mathbb{1}_{\llbracket 0, \tau \wedge \tau^{\prime} \wedge \rho_{k} \rrbracket} \mathbb{1}_{\left\{y_{0}=y_{0}^{\prime}\right\}}=Y^{\prime} \mathbb{1}_{\llbracket 0, \tau \wedge \tau^{\prime} \wedge \rho_{k} \rrbracket} \mathbb{1}_{\left\{y_{0}=y_{0}^{\prime}\right\}} \quad \forall k \in \mathbb{N}_{0} .
$$

The identity (3.33) holds true for $k=0$, because by Lemma 2.3, we have $\varphi_{0}=0$.

For the induction step $k \rightarrow k+1$, we suppose that identity (3.33) is satisfied. We define the stopping time $\tau_{k}:=\tau \wedge \tau^{\prime} \wedge \varrho_{k+1}$ and the set $\Gamma_{k}:=\left\{\varrho_{k} \leq \tau_{k}\right\} \cap\left\{y_{0}=y_{0}^{\prime}\right\} \in \mathcal{F}_{\rho_{k}}$. By Lemma 3.7, the processes $Y^{\left(\rho_{k}, \Gamma_{k}\right)}:=Y_{\rho_{k}+\bullet} \mathbb{1}_{\Gamma_{k}}$ and $Y^{\prime}\left(\rho_{k}, \Gamma_{k}\right):=Y_{\rho_{k} \cdot \bullet}^{\prime} \mathbb{1}_{\Gamma_{k}}$ defined according to (3.11) are $\mathbb{F}^{\left(\rho_{k}\right)_{-}}$ adapted local strong solutions to (1.4) with parameters (3.12), where $\tau=\rho_{k}$ and $\Gamma=\Gamma_{k}$, initial conditions $Y_{\rho_{k}} \mathbb{1}_{\Gamma_{k}}$ and $Y_{\rho_{k}}^{\prime} \mathbb{1}_{\Gamma_{k}}$, and lifetime $\left(\tau_{k}-\rho_{k}\right)^{+}$.

Let $n \in \mathbb{N}$ be arbitrary, and set $\Gamma_{k n}:=\Gamma_{k} \cap\left\{\rho_{k} \leq n\right\} \in \mathcal{F}_{\rho_{k}}$. The processes $Y^{\left(\rho_{k}, \Gamma_{k n}\right)}:=Y^{\left(\rho_{k}, \Gamma_{k}\right)} \mathbb{1}_{\Gamma_{k n}}$ and $Y^{\left(\varphi_{k}, \Gamma_{k n}\right)}:=Y^{\prime\left(\rho_{k}, \Gamma_{k}\right)} \mathbb{1}_{\Gamma_{k n}}$ are $\mathbb{F}^{\left(\rho_{k}\right)}$-adapted local strong solutions to (1.4) with parameters (3.25), where $\Gamma=\Gamma_{k n}$, initial conditions $Y_{\rho_{k}} \mathbb{1}_{\Gamma_{k n}}$ and $Y_{\rho_{k}}^{\prime} \mathbb{1}_{\Gamma_{k n}}$, and lifetime $\left(\tau_{k}-\rho_{k}\right)^{+}$. By Lemma 3.9, the processes $Y^{\left(\rho_{k}, \Gamma_{k n}\right)-}$ and $Y^{\left(\rho_{k}, \Gamma_{k n}\right)-}$ defined according to (3.26) are $\mathbb{F}^{\left(\rho_{k}\right)}$-adapted local strong solutions to (1.5) with parameters (3.25), where $\Gamma=\Gamma_{k n}$, initial conditions $Y_{\rho_{k}} \mathbb{1}_{\Gamma_{k n}}$ and $Y_{\rho_{k}}^{\prime} \mathbb{1}_{\Gamma_{k n}}$, and lifetime $\left(\tau_{k}-\rho_{k}\right)^{+}$. According to Lemma 3.6, the mappings $\left(a^{\left(\rho_{k}, \Gamma_{k n}\right)}, b^{\left(\rho_{k}, \Gamma_{k n}\right)},\left.c^{\left(\rho_{k}, \Gamma_{k n}\right)}\right|_{B}\right)$ are locally Lipschitz, too. Therefore, by Proposition 3.11, we have up to indistinguishability

$$
Y^{\left(\rho_{k}, \Gamma_{k n}\right){ }^{-}} \mathbb{1}_{\llbracket 0,\left(\tau_{k}-\rho_{k}\right)^{+} \rrbracket}=Y^{\left(\rho_{k}, \Gamma_{k n}\right)-} \mathbb{1}_{\llbracket 0,\left(\tau_{k}-\rho_{k}\right)^{+} \rrbracket} \quad \forall n \in \mathbb{N} .
$$

By the Definition (3.26), we deduce that up to indistinguishability

$$
Y^{\left(\rho_{k}, \Gamma_{k n}\right)} \mathbb{1}_{\llbracket 0,\left(\tau_{k}-\rho_{k}\right)^{+} \rrbracket}=Y^{\prime\left(\varphi_{k}, \Gamma_{k n}\right)} \mathbb{1}_{\llbracket 0,\left(\tau_{k}-\rho_{k}\right)^{+} \rrbracket} \quad \forall n \in \mathbb{N},
$$

and hence, we have up to indistinguishability

$$
Y^{\left(\rho_{k}, \Gamma_{k}\right)} \mathbb{1}_{\left\{\rho_{k} \leq n\right\}} \mathbb{1}_{\llbracket 0,\left(\tau_{k}-\rho_{k}\right)^{+} \rrbracket}=Y^{\left(\varphi_{k}, \Gamma_{k}\right)} \mathbb{1}_{\left\{\rho_{k} \leq n\right\}} \mathbb{1}_{\llbracket 0,\left(\tau_{k}-\rho_{k}\right)^{+} \rrbracket} \quad \forall n \in \mathbb{N} .
$$

By Lemma 2.3, we have $\mathbb{P}\left(\rho_{k}<\infty\right)=1$, and hence, we get up to indistinguishability

$$
Y^{\left(\rho_{k}, \Gamma_{k}\right)} \mathbb{1}_{\llbracket 0,\left(\tau_{k}-\rho_{k}\right)^{+} \rrbracket}=Y^{\prime\left(\rho_{k}, \Gamma_{k}\right)} \mathbb{1}_{\llbracket 0,\left(\tau_{k}-\rho_{k}\right)^{+} \rrbracket} .
$$

Therefore, we have up to indistinguishability

$$
Y_{\rho_{k}+\bullet} \mathbb{1}_{\left\{\rho_{k} \leq \tau_{k}\right\}} \mathbb{1}_{\llbracket 0,\left(\tau_{k}-\rho_{k}\right)^{+} \rrbracket} \mathbb{1}_{\left\{y_{0}=y_{0}^{\prime}\right\}}=Y_{\rho_{k}+\bullet}^{\prime} \mathbb{1}_{\left\{\rho_{k} \leq \tau_{k}\right\}} \mathbb{1}_{\llbracket 0,\left(\tau_{k}-\rho_{k}\right)^{+} \rrbracket} \mathbb{1}_{\left\{y_{0}=y_{0}^{\prime}\right\}}
$$

Consequently, we have up to indistinguishability

$$
Y \mathbb{1}_{\left\{\rho_{k} \leq \tau_{k}\right\}} \mathbb{1}_{\llbracket \rho_{k}, \tau_{k} \rrbracket} \mathbb{1}_{\left\{y_{0}=y_{0}^{\prime}\right\}}=Y^{\prime} \mathbb{1}_{\left\{\rho_{k} \leq \tau_{k}\right\}} \mathbb{1}_{\llbracket \rho_{k}, \tau_{k} \rrbracket} \mathbb{1}_{\left\{y_{0}=y_{0}^{\prime}\right\}} .
$$


Together with the induction hypothesis, it follows that

$$
Y \mathbb{1}_{\llbracket 0, \tau_{k} \rrbracket} \mathbb{1}_{\left\{y_{0}=y_{0}^{\prime}\right\}}=Y^{\prime} \mathbb{1}_{\llbracket 0, \tau_{k} \rrbracket} \mathbb{1}_{\left\{y_{0}=y_{0}^{\prime}\right\}}
$$

which establishes (3.33). Since by Lemma 2.3, that we have $\mathbb{P}\left(\rho_{k} \rightarrow \infty\right)=1$, we deduce

$$
Y^{\tau \wedge \tau^{\prime}} \mathbb{1}_{\left\{y_{0}=y_{0}^{\prime}\right\}}=\left(Y^{\prime}\right)^{\tau \wedge \tau^{\prime}} \mathbb{1}_{\left\{y_{0}=y_{0}^{\prime}\right\}},
$$

completing the proof.

\subsection{Existence of Strong Solutions to Hilbert Space-Valued SDEs}

Now, we will deal with the existence of strong solutions to the SDE (1.4).

Proposition 3.13. One supposes that the mappings $\left(a, b,\left.c\right|_{B}\right)$ are locally Lipschitz and satisfy the linear growth condition. Then, existence of strong solutions to (1.5) holds.

Proof. If the mappings $\left(a, b,\left.c\right|_{B}\right)$ are Lipschitz continuous, then we have existence and uniqueness of strong solutions to (1.5) for every initial condition $y_{0} \in \mathcal{L}^{2}\left(\mathcal{F}_{0} ; \mathscr{d}\right)$, see, for example, [6, Corollary 10.3].

For $\left(a, b,\left.c\right|_{B}\right)$ being locally Lipschitz and satisfying the linear growth condition, for any initial condition $y_{0} \in \mathcal{L}^{2}\left(\mathcal{F}_{0} ; \mathscr{d}\right)$, we adopt the technique from the proof of $[21$, Theorem 4.11]. For $k \in \mathbb{N}$, we define the retraction

$$
R_{k}: \mathscr{H} \longrightarrow \mathscr{L}, \quad R_{k}(y):= \begin{cases}y & \text { if }\|y\| \leq k, \\ k \frac{y}{\|y\|} & \text { if }\|y\|>k\end{cases}
$$

and the mappings $a_{k}: \Omega \times \mathbb{R}_{+} \times \mathscr{H} \rightarrow \mathscr{H}, b_{k}: \Omega \times \mathbb{R}_{+} \times \mathscr{H} \rightarrow L_{2}^{0}(\mathscr{H})$, and $c_{k}: \Omega \times \mathbb{R}_{+} \times$ $\mathscr{d} \times E \rightarrow \mathscr{d}$ as

$$
a_{k}:=a \circ R_{k}, \quad b_{k}:=b \circ R_{k}, \quad c_{k}(\bullet, x):=c(\bullet, x) \circ R_{k} .
$$

These mappings are Lipschitz continuous, and hence, there exists a strong solution $Y^{(k)}$ to the SDE (1.5) with parameters $a=a_{k}, b=b_{k}$, and $c=c_{k}$, and initial condition $y_{0}$. Using the linear growth condition, Gronwall's lemma, and Doob's martingale inequality, we can show that $\mathbb{P}\left(\tau_{k} \rightarrow \infty\right)=1$, where

$$
\tau_{k}:=\inf \left\{t \geq 0:\left\|Y_{t}^{(k)}\right\|>k\right\}, \quad k \in \mathbb{N}_{0}
$$


that is, the solutions do not explode. Consequently, the process

$$
Y:=y_{0} \mathbb{1}_{\llbracket \tau_{0} \rrbracket}+\sum_{k \in \mathbb{N}} Y^{(k)} \mathbb{1}_{\rrbracket \tau_{k-1}, \tau_{k} \rrbracket}
$$

is a strong solution to (1.5) with initial condition $y_{0}$.

Finally, for a general $\mathcal{F}_{0}$-measurable initial condition $y_{0}: \Omega \rightarrow \mathscr{H}$, the process $Y:=$ $\sum_{k \in \mathbb{N}} Y^{(k)} \mathbb{1}_{\Omega_{k}}$ is a strong solution to (1.5) with initial condition $y_{0}$, where $\left(\Omega_{k}\right)_{k \in \mathbb{N}} \subset \mathcal{F}_{0}$ denotes the partition of $\Omega$ given by $\Omega_{k}:=\left\{\left\|y_{0}\right\| \in[k-1, k)\right\}$, and where for each $k \in \mathbb{N}$ the process $Y^{(k)}$ denotes a strong solution to (1.5) with initial condition $y_{0} \mathbb{1}_{\Omega_{k}}$.

Theorem 3.14. One supposes that the mappings $\left(a, b,\left.c\right|_{B}\right)$ are locally Lipschitz and satisfy the linear growth condition. Then, existence of strong solutions to (1.4) holds.

Proof. Let $y_{0}: \Omega \rightarrow \mathscr{H}$ be an arbitrary $\mathcal{F}_{0}$-measurable random variable. By induction, we will prove that for each $k \in \mathbb{N}_{0}$ there exists a local strong solution $Y^{(k)}$ to (1.4) with initial condition $y_{0}$ and lifetime $\rho_{k}$. By Lemma 2.3, we have $\rho_{0}=0$, providing the assertion for $k=0$.

For the induction step $k \rightarrow k+1$, let $Y^{(k)}$ be a local strong solution to (1.4) with initial condition $y_{0}$ and lifetime $\rho_{k}$. Let $n \in \mathbb{N}$ be arbitrary, and set $\Gamma_{k n}:=\left\{\varrho_{k} \in[n-1, n)\right\} \in \mathcal{F}_{\rho_{k}}$. By Lemma 3.6, the mappings $\left(a^{\left(\rho_{k}, \Gamma_{k n}\right)}, b^{\left(\rho_{k}, \Gamma_{k n}\right)},\left.c^{\left(\rho_{k}, \Gamma_{k n}\right)}\right|_{B}\right)$ are locally Lipschitz, too. Therefore, by Proposition 3.13, there exists an $\mathbb{F}^{\left(\rho_{k}\right)}$-adapted strong solution $Y^{\left(\rho_{k}, \Gamma_{k n}\right)-}$ to (1.5) with parameters (3.25), where $\Gamma=\Gamma_{k n}$, and initial condition $Y_{\rho_{k}}^{(k)} \mathbb{1}_{\Gamma_{k n}}$. By Lemma 3.10, the process $Y^{\left(Q_{k}, \Gamma_{k n}\right)}$ defined according to (3.32) is an $\mathbb{F}^{\left(\rho_{k}\right)}$-adapted local strong solution to (1.4) with parameters (3.25), where $\Gamma=\Gamma_{k n}$, initial condition $Y_{\rho_{k}}^{(k)} \mathbb{1}_{\Gamma_{k n}}$, and lifetime $\rho_{k+1}-\rho_{k}$. Noting that $\left(\Gamma_{k n}\right)_{n \in \mathbb{N}}$ is a partition of $\Omega$, it follows that $Y^{\left(\rho_{k}\right)}:=\sum_{n \in \mathbb{N}} Y^{\left(\rho_{k}, \Gamma_{k n}\right)}$ is an $\mathbb{F}^{\left(\rho_{k}\right)}$-adapted local strong solution to (1.4) with initial condition $Y_{\rho_{k}}^{(k)}$ and lifetime $\rho_{k+1}-\rho_{k}$. By Lemma 3.8, the process

$$
Y^{(k+1)}:=Y^{(k)} \mathbb{1}_{\llbracket 0, \rho_{k} \rrbracket}+Y_{\bullet-\rho_{k}}^{\left(\rho_{k}\right)} \mathbb{1}_{\rrbracket \rho_{k}, \rho_{k+1} \rrbracket}
$$

defined according to (3.17) is an $\mathbb{F}$-adapted local strong solution to (1.4) with initial condition $y_{0}$ and lifetime $\varphi_{k+1}$.

Consequently, for each $k \in \mathbb{N}_{0}$, there exists a local strong solution $Y^{(k)}$ to (1.4) with initial condition $y_{0}$ and lifetime $\rho_{k}$. By Lemma 2.3, we have $\mathbb{P}\left(\rho_{k} \rightarrow \infty\right)=1$. Hence, it follows that

$$
Y:=y_{0} \mathbb{1}_{\llbracket \rho_{0} \rrbracket}+\sum_{k \in \mathbb{N}} Y^{(k)} \mathbb{1}_{\rrbracket \rho_{k-1}, \rho_{k} \rrbracket}
$$

is an $\mathbb{F}$-adapted strong solution to (1.4) with initial condition $y_{0}$.

Theorem 3.15. One supposes that the mappings $\left(a, b,\left.c\right|_{B}\right)$ are locally Lipschitz and locally bounded. Then, existence of local strong solutions to (1.4) holds.

Proof. Let $y_{0}: \Omega \rightarrow \mathscr{H}$ be an arbitrary $\mathcal{F}_{0}$-measurable random variable. We define the partition $\left(\Omega_{k}\right)_{k \in \mathbb{N}} \subset \mathcal{F}_{0}$ of $\Omega$ by $\Omega_{k}:=\left\{\left\|y_{0}\right\| \in[k-1, k)\right\}$. Furthermore, for each $k \in$ $\mathbb{N}$, we define the mappings $a_{k}: \Omega \times \mathbb{R}_{+} \times \mathscr{d} \rightarrow \mathscr{d}, b_{k}: \Omega \times \mathbb{R}_{+} \times \mathscr{d} \rightarrow L_{2}^{0}(\mathscr{l})$, 
and $c_{k}: \Omega \times \mathbb{R}_{+} \times \mathscr{H} \times E \rightarrow \mathscr{H}$ as in the proof of Proposition 3.13. These mappings are locally Lipschitz and satisfy the linear growth condition. By Theorem 3.14, there exists a strong solution $Y^{(k)}$ to (1.4) with parameters $a=a_{k}, b=b_{k}$, and $c=c_{k}$, and initial condition $y_{0} \mathbb{1}_{\Omega_{k}}$. The stopping time

$$
\tau_{k}:=\inf \left\{t \geq 0:\left\|Y_{t}^{(k)}\right\|>k\right\}
$$

is strictly positive, and $Y^{(k)}$ is a local strong solution to (1.4) with initial condition $y_{0} \mathbb{1}_{\Omega_{k}}$ and lifetime $\tau_{k}$. The stopping time $\tau:=\sum_{k \in \mathbb{N}} \tau_{k} \mathbb{1}_{\Omega_{k}}$ is strictly positive, and the process $Y:=$ $\sum_{k \in \mathbb{N}} Y^{(k)} \mathbb{1}_{\Omega_{k}}$ is a local strong solution to (1.4) with initial condition $y_{0}$ and lifetime $\tau$.

\subsection{Comparison with the Method of Successive Approximations}

So far, our investigations provide the following result concerning existence and uniqueness of global strong solutions to the SDE (1.4).

Theorem 3.16. If $\left(a, b,\left.c\right|_{B}\right)$ are locally Lipschitz and satisfy the linear growth condition, then existence and uniqueness of strong solutions to (1.4) hold.

Proof. This is a direct consequence of Theorems 3.12 and 3.14 .

Now, we will provide a comparison with [14], where the authors also study Hilbert space-valued SDEs of the type (1.4). Their result [14, Theorem 2.1] is based on the method of successive approximations (see also $[22,23]$ ) and considerably goes beyond the classical global Lipschitz conditions. For the sake of simplicity, let us recall the required assumptions in the time-homogeneous Markovian framework. In order to apply [14, Theorem 2.1], for some constant $p \geq 2$, we need the estimate

$$
\begin{gathered}
\left\|a\left(y_{1}\right)-a\left(y_{2}\right)\right\|^{p}+\left\|b\left(y_{1}\right)-b\left(y_{2}\right)\right\|_{L_{2}^{0}(\mathscr{d})}^{p}+\int_{B}\left\|c\left(y_{1}, x\right)-c\left(y_{2}, y\right)\right\|^{p} F(d x) \\
+\left(\int_{B}\left\|c\left(y_{1}, x\right)-c\left(y_{2}, x\right)\right\|^{2} F(d x)\right)^{p / 2} \leq \kappa\left(\left\|y_{1}-y_{2}\right\|^{p}\right) \quad \forall y_{1}, y_{2} \in \mathscr{H},
\end{gathered}
$$

where $\mathcal{\kappa}: \mathbb{R}_{+} \rightarrow \mathbb{R}_{+}$denotes a continuous, nondecreasing function with $\mathcal{\kappa}(0)=0$, and further conditions, which are precisely stated in [14], must be fulfilled. These conditions are satisfied if $\mathcal{K}$ is a continuous, nondecreasing, and concave function such that

$$
\int_{0}^{\epsilon} \frac{1}{\kappa(u)} d u=\infty \text { for each } \epsilon>0
$$

In particular, we may choose $\kappa(u)=u$ for $u \in \mathbb{R}_{+}$, and consequently, both results, Theorem 3.16 and [14, Theorem 2.1], cover the classical situation, where global Lipschitz conditions are imposed.

However, there are situations where [14, Theorem 2.1] can be applied, while Theorem 3.16 does not apply, and vice versa. For the sake of simplicity, in the following two examples, we assume that $\mathscr{H}=\mathbb{R}$ and $b \equiv c \equiv 0$. 
Example 3.17. We fix an arbitrary constant $0<\delta<\exp (-1)$ and define the functions $\kappa, \rho$ : $\mathbb{R}_{+} \rightarrow \mathbb{R}_{+}$by

$$
\kappa(u):= \begin{cases}0, & u=0, \\ -u \ln u, & 0<u<\delta, \\ -\delta \ln \delta-(1+\ln \delta)(u-\delta) & u \geq \delta,\end{cases}
$$

as well as

$$
\rho(u):= \begin{cases}0, & u=0, \\ u \sqrt{-\ln \left(u^{2}\right)}, & 0<u<\sqrt{\delta}, \\ \sqrt{-\delta \ln \delta-(1+\ln \delta)\left(u^{2}-\delta\right)} & u \geq \sqrt{\delta},\end{cases}
$$

compare with [22, Remark 1$]$. Let $a: \mathbb{R} \rightarrow \mathbb{R}$ be a mapping such that

$$
\left|a\left(y_{1}\right)-a\left(y_{2}\right)\right| \leq \rho\left(\left|y_{1}-y_{2}\right|\right) \quad \forall y_{1}, y_{2} \in \mathbb{R} \text {. }
$$

Then we have the estimate

$$
\left|a\left(y_{1}\right)-a\left(y_{2}\right)\right|^{2} \leq \kappa\left(\left|y_{1}-y_{2}\right|^{2}\right) \quad \forall y_{1}, y_{2} \in \mathbb{R}
$$

showing that condition (3.49) with $p=2$ is satisfied. Moreover, $\kappa$ is a continuous, nondecreasing, concave function, and condition (3.50) is satisfied, because for each $0<\epsilon<\delta$, we have

$$
\int_{0}^{\epsilon} \frac{1}{\kappa(u)} d u=-\int_{0}^{\epsilon} \frac{1}{u \ln u} d u=-\left.\ln |\ln u|\right|_{u=0} ^{u=e}=-\ln |\ln \epsilon|+\lim _{u \rightarrow 0} \ln |\ln u|=\infty
$$

Consequently, [14, Theorem 2.1] applies. However, we have

$$
\rho^{\prime}(u)=\sqrt{-\ln \left(u^{2}\right)}-\frac{1}{\sqrt{-\ln \left(u^{2}\right)}} \text { for } u \in(0, \sqrt{\delta}),
$$

and thus, $\lim _{u \rightarrow 0} \rho^{\prime}(u)=\infty$. Therefore, the mapping $a: \mathbb{R} \rightarrow \mathbb{R}$ might fail to be locally Lipschitz, and hence, Theorem 3.16 does not apply.

Example 3.18. Let us define the mapping $a: \mathbb{R} \rightarrow \mathbb{R}$ as follows. For $n \in \mathbb{N}_{0}$, we define $a$ on the interval $[n, n+1]$ by

$$
a(y):= \begin{cases}n, & y \in\left[n, n+1-\frac{1}{n+1}\right], \\ n+(n+1)\left(y-\left(n+1-\frac{1}{n+1}\right)\right), & y \in\left[n+1-\frac{1}{n+1}, n+1\right] .\end{cases}
$$


This defines the mapping $a: \mathbb{R}_{+} \rightarrow \mathbb{R}$, which we extend to a mapping $a: \mathbb{R} \rightarrow \mathbb{R}$ by symmetry

$$
a(y):=a(-y), \quad y \in \mathbb{R}_{-}
$$

Then, $a$ is locally Lipschitz and satisfies the linear growth condition, and hence, Theorem 3.16 applies. However, there are no constant $p \geq 2$ and no continuous, nondecreasing function $\kappa: \mathbb{R}_{+} \rightarrow \mathbb{R}_{+}$with $\kappa(0)=0$ such that

$$
\left|a\left(y_{1}\right)-a\left(y_{2}\right)\right|^{p} \leq \kappa\left(\left|y_{1}-y_{2}\right|^{p}\right) \quad \forall y_{1}, y_{2} \in \mathbb{R}
$$

Suppose, on the contrary, that there exists a continuous, nondecreasing function $\kappa: \mathbb{R}_{+} \rightarrow \mathbb{R}_{+}$ with $\kappa(0)=0$ fulfilling (3.59). Then we have

$$
|\kappa(u)| \geq 1 \quad \forall u \in(0,1]
$$

Indeed, let $u \in(0,1]$ be arbitrary. Then, there exists $n \in \mathbb{N}$ with $1 / n \leq u$. Moreover, by the definition of the mapping $a: \mathbb{R} \rightarrow \mathbb{R}$, there are $y_{1}, y_{2} \in \mathbb{R}$ such that

$$
\left|y_{1}-y_{2}\right| \leq\left(\frac{1}{n}\right)^{1 / p}, \quad\left|a\left(y_{1}\right)-a\left(y_{2}\right)\right|=1
$$

Therefore, using the monotonicity of $\kappa$ and (3.59), we obtain

$$
\kappa(u) \geq \kappa\left(\frac{1}{n}\right) \geq \kappa\left(\left|y_{1}-y_{2}\right|^{p}\right) \geq\left|a\left(y_{1}\right)-a\left(y_{2}\right)\right|^{p}=1
$$

showing (3.60). Now, the continuity of $\kappa$ yields the contradiction $\kappa(0) \geq 1$. Consequently, condition (3.49) is not satisfied, and thus, we cannot use [14, Theorem 2.1] in this case.

\section{Existence and Uniqueness of Mild Solutions to Hilbert Space-Valued SPDEs}

In this section, we establish existence and uniqueness of (local) mild solutions to Hilbert space-valued SPDEs of the type (1.3).

Let $H$ be a separable Hilbert space, let $\left(S_{t}\right)_{t \geq 0}$ be a $C_{0}$-semigroup on $H$ with infinitesimal generator $A: \Phi(A) \subset H \rightarrow H$, and let $B \in \mathcal{E}$ be a set with $F\left(B^{c}\right)<\infty$. Furthermore, let $\alpha: \Omega \times \mathbb{R}_{+} \times H \rightarrow H$ and $\sigma: \Omega \times \mathbb{R}_{+} \times H \rightarrow L_{2}^{0}(H)$ be $D \otimes B(H)$-measurable mappings, and let $\gamma: \Omega \times \mathbb{R}_{+} \times H \times E \rightarrow H$ be a $D \otimes \mathbb{B}(H) \otimes \mathcal{E}$-measurable mapping. 
Throughout this section, we suppose that there exist another separable Hilbert space $\mathscr{H}$, a $C_{0}$-group $\left(U_{t}\right)_{t \in \mathbb{R}}$ on $\mathscr{l}$, and continuous linear operators $\ell \in L(H, \mathscr{l}), \pi \in L(\mathscr{l}, H)$ such that the diagram

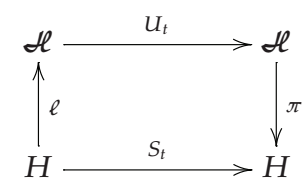

commutes for every $t \in \mathbb{R}_{+}$, that is,

$$
\pi U_{t} \ell=S_{t} \quad \forall t \in \mathbb{R}_{+} .
$$

Remark 4.1. According to [6, Proposition 8.7], this assumption is satisfied if the semigroup $\left(S_{t}\right)_{t \geq 0}$ is pseudocontractive (one also uses the notion quasicontractive), that is, there is a constant $\omega \in \mathbb{R}$ such that

$$
\left\|S_{t}\right\| \leq e^{\omega t} \quad \forall t \geq 0
$$

This result relies on the Szökefalvi-Nagy theorem on unitary dilations (see, e.g., [24, Theorem I.8.1] or [25, Section 7.2]). In the spirit of [24], the group $\left(U_{t}\right)_{t \in \mathbb{R}}$ is called a dilation of the semigroup $\left(S_{t}\right)_{t \geq 0}$.

Remark 4.2. The Szökefalvi-Nagy theorem was also utilized in $[26,27]$ in order to establish results concerning stochastic convolution integrals.

Now, we define the mappings $a: \Omega \times \mathbb{R}_{+} \times \mathscr{H} \rightarrow \mathscr{H}, b: \Omega \times \mathbb{R}_{+} \times \mathscr{H} \rightarrow L_{2}^{0}(\mathscr{H})$, and $c: \Omega \times \mathbb{R}_{+} \times \mathscr{L} \times E \rightarrow \mathscr{d}$ by

$$
\begin{gathered}
a(t, y):=U_{-t} \ell \alpha\left(t, \pi U_{t} y\right), \\
b(t, y):=U_{-t} \ell \sigma\left(t, \pi U_{t} y\right), \\
c(t, y, x):=U_{-t} \ell \gamma\left(t, \pi U_{t} y, x\right) .
\end{gathered}
$$

Note that $a$ and $b$ are $D \otimes B(\mathscr{\ell})$-measurable and that $c$ is $D \otimes B(\mathscr{\ell}) \otimes \mathcal{E}$-measurable.

Lemma 4.3. The following statements are true:

(1) if $\left(\alpha, \sigma,\left.\gamma\right|_{B}\right)$ are locally Lipschitz, then $\left(a, b,\left.c\right|_{B}\right)$ are locally Lipschitz, too;

(2) if $\left(\alpha, \sigma,\left.\gamma\right|_{B}\right)$ satisfy the linear growth condition, then $\left(a, b,\left.c\right|_{B}\right)$ satisfy the linear growth condition, too;

(3) if $\left(\alpha, \sigma,\left.\gamma\right|_{B}\right)$ are locally bounded, then $\left(a, b,\left.c\right|_{B}\right)$ are locally bounded, too.

Proof. All three statements are straightforward to check. 
International Journal of Stochastic Analysis

Proposition 4.4. Let $z_{0}: \Omega \rightarrow H$ be a $\mathcal{F}_{0}$-measurable random variable, and let $\tau$ be a stopping time. Then, the following statements are true:

(1) if $Y$ is a local strong solution to (1.4) with initial condition $\ell z_{0}$ and lifetime $\tau$, then $Z:=$ $\pi U Y$ is a local mild solution to (1.3) with initial condition $z_{0}$ and lifetime $\tau$;

(2) if $Z$ is a local mild solution to (1.3) with initial condition $z_{0}$ and lifetime $\tau$, then the process $Y$ defined as

$$
\begin{aligned}
Y_{t}:= & \ell z_{0}+\int_{0}^{t \wedge \tau} U_{-s} \ell \alpha\left(s, Z_{s}\right) d s+\int_{0}^{t \wedge \tau} U_{-s} \ell \sigma\left(s, Z_{s}\right) d W_{s} \\
& +\int_{0}^{t \wedge \tau} \int_{B} U_{-s} \lg \left(s, Z_{s-} x\right)(\mu(d s, d x)-F(d x) d s) \\
& +\int_{0}^{t \wedge \tau} \int_{B^{c}} U_{-s} \operatorname{lr}\left(s, Z_{s-}, x\right) \mu(d s, d x), \quad t \geq 0
\end{aligned}
$$

is a local strong solution to (1.4) with initial condition $\ell z_{0}$ and lifetime $\tau$, and one has $Z^{\tau}=\pi U Y^{\tau}$.

Proof. Let $Y$ be a local strong solution to (1.4) with initial condition $\ell z_{0}$ and lifetime $\tau$. Then we have

$$
\begin{aligned}
Z_{t \wedge \tau}= & \pi U_{t \wedge \tau} Y_{t \wedge \tau} \\
= & \pi U_{t \wedge \tau}\left(\ell z_{0}+\int_{0}^{t \wedge \tau} a\left(s, Y_{s}\right) d s+\int_{0}^{t \wedge \tau} b\left(s, Y_{s}\right) d W_{s}\right. \\
& \left.\quad+\int_{0}^{t \wedge \tau} \int_{B} c\left(s, Y_{s-}, x\right)(\mu(d s, d x)-F(d x) d s)+\int_{0}^{t \wedge \tau} \int_{B^{c}} c\left(s, Y_{s-}, x\right) \mu(d s, d x)\right) .
\end{aligned}
$$

By the Definitions (4.4) of $a, b, c$, we obtain

$$
\begin{aligned}
Z_{t \wedge \tau}=\pi U_{t \wedge \tau}\left(\ell z_{0}+\int_{0}^{t \wedge \tau} U_{-s} \ell \alpha\left(s, \pi U_{s} Y_{s}\right) d s+\int_{0}^{t \wedge \tau} U_{-s} \ell \sigma\left(s, \pi U_{s} Y_{s}\right) d W_{s}\right. \\
\quad+\int_{0}^{t \wedge \tau} \int_{B} U_{-s} \ell \gamma\left(s, \pi U_{s} Y_{s-} x\right)(\mu(d s, d x)-F(d x) d s) \\
\left.+\int_{0}^{t \wedge \tau} \int_{B^{c}} U_{-s} \ell \gamma\left(s, \pi U_{s} Y_{s-}, x\right) \mu(d s, d x)\right) .
\end{aligned}
$$


Therefore, by (4.2), and since $Z=\pi U Y$, we arrive at

$$
\begin{aligned}
Z_{t \wedge \tau}= & S_{t \wedge \tau} Z_{0}+\int_{0}^{t \wedge \tau} S_{(t \wedge \tau)-s} \alpha\left(s, Z_{s}\right) d s+\int_{0}^{t \wedge \tau} S_{(t \wedge \tau)-s} \sigma\left(s, Z_{s}\right) d W_{s} \\
& +\int_{0}^{t \wedge \tau} \int_{B} S_{(t \wedge \tau)-s} \gamma\left(s, Z_{s-}, x\right)(\mu(d s, d x)-F(d x) d s) \\
& +\int_{0}^{t \wedge \tau} \int_{B^{c}} S_{(t \wedge \tau)-s} \gamma\left(s, Z_{s-}, x\right) \mu(d s, d x),
\end{aligned}
$$

showing that $Z$ is a local mild solution to (1.3) with initial condition $z_{0}$ and lifetime $\tau$. This establishes the first statement. Now, let $Z$ be a local mild solution to (1.3) with initial condition $z_{0}$ and lifetime $\tau$. Then we have (4.8), and therefore, by (4.2) and the Definition (4.5) of $Y$, we obtain

$$
\begin{aligned}
Z_{t \wedge \tau}=\pi U_{t \wedge \tau}\left(\ell z_{0}+\int_{0}^{t \wedge \tau} U_{-s} \ell \alpha\left(s, Z_{s}\right) d s+\int_{0}^{t \wedge \tau} U_{-s} \ell \sigma\left(s, Z_{s}\right) d W_{s}\right. \\
\quad+\int_{0}^{t \wedge \tau} \int_{B} U_{-s} \operatorname{lr}\left(s, Z_{s^{-}} x\right)(\mu(d s, d x)-F(d x) d s) \\
\left.\quad+\int_{0}^{t \wedge \tau} \int_{B^{c}} U_{-s} \operatorname{lr}\left(s, Z_{s-}, x\right) \mu(d s, d x)\right)=\pi U_{t \wedge \tau} Y_{t \wedge \tau}
\end{aligned}
$$

showing that $Z^{\tau}=\pi U Y^{\tau}$. Therefore, by the Definition (4.5) of $Y$, we obtain

$$
\begin{aligned}
Y_{t \wedge \tau}= & \ell z_{0}+\int_{0}^{t \wedge \tau} U_{-s} \ell \alpha\left(s, \pi U_{s} Y_{s}\right) d s+\int_{0}^{t \wedge \tau} U_{-s} \ell \sigma\left(s, \pi U_{s} Y_{s}\right) d W_{s} \\
& +\int_{0}^{t \wedge \tau} \int_{B} U_{-s} \ell \gamma\left(s, \pi U_{s} Y_{s-}, x\right)(\mu(d s, d x)-F(d x) d s) \\
& +\int_{0}^{t \wedge \tau} \int_{B^{c}} U_{-s} \ell \gamma\left(s, \pi U_{s} Y_{s-}, x\right) \mu(d s, d x) .
\end{aligned}
$$

Taking into account the Definitions (4.4) of $a, b, c$, we get

$$
\begin{aligned}
Y_{t \wedge \tau}= & \ell z_{0}+\int_{0}^{t \wedge \tau} a\left(s, Y_{s}\right) d s+\int_{0}^{t \wedge \tau} b\left(s, Y_{s}\right) d W_{s} \\
& +\int_{0}^{t \wedge \tau} \int_{B} c\left(s, Y_{s^{-}}, x\right)(\mu(d s, d x)-F(d x) d s)+\int_{0}^{t \wedge \tau} \int_{B^{c}} c\left(s, Y_{s^{-}}, x\right) \mu(d s, d x),
\end{aligned}
$$

showing that $Y$ is a local strong solution to (1.4) with initial condition $\ell z_{0}$ and lifetime $\tau$. 
Theorem 4.5. The following statements are true:

(1) if $\left(\alpha, \sigma,\left.\gamma\right|_{B}\right)$ are locally Lipschitz and satisfy the linear growth condition, then existence and uniqueness of mild solutions to (1.3) hold;

(2) if $\left(\alpha, \sigma,\left.\gamma\right|_{B}\right)$ are locally Lipschitz and locally bounded, then existence and uniqueness of local mild solutions to (1.3) hold;

(3) if $\left(\alpha, \sigma,\left.\gamma\right|_{B}\right)$ are locally Lipschitz, then uniqueness of local mild solutions to (1.3) holds.

Proof. Suppose that $\left(\alpha, \sigma,\left.\gamma\right|_{B}\right)$ are locally Lipschitz. Let $Z$ and $Z^{\prime}$ be two local mild solutions to (1.3) with initial conditions $z_{0}$ and $z_{0}^{\prime}$ and lifetimes $\tau$ and $\tau^{\prime}$. We define the $\mathscr{d}$-valued processes $Y$ and $Y^{\prime}$ according to (4.5). By Proposition 4.4, the processes $Y$ and $Y^{\prime}$ are local strong solutions to (1.4) with initial conditions $\ell z_{0}$ and $\ell z_{0}^{\prime}$ and lifetimes $\tau$ and $\tau^{\prime}$, and we have $Z^{\tau}=\pi U Y^{\tau}$ and $\left(Z^{\prime}\right)^{\tau^{\prime}}=\pi U\left(Y^{\prime}\right)^{\tau^{\prime}}$. By Lemma 4.3, the mappings $\left(a, b,\left.c\right|_{B}\right)$ are also locally Lipschitz, and hence, Theorem 3.12 yields that up to indistinguishability

$$
Y^{\tau \wedge \tau^{\prime}} \mathbb{1}_{\left\{\ell z_{0}=\ell z_{0}^{\prime}\right\}}=\left(Y^{\prime}\right)^{\tau \wedge \tau^{\prime}} \mathbb{1}_{\left\{\ell z_{0}=\ell z_{0}^{\prime}\right\}}
$$

Therefore, we have up to indistinguishability

$$
Z^{\tau \wedge \tau^{\prime}} \mathbb{1}_{\left\{z_{0}=z_{0}^{\prime}\right\}}=\pi U Y^{\tau \wedge \tau^{\prime}} \mathbb{1}_{\left\{z_{0}=z_{0}^{\prime}\right\}}=\pi U\left(Y^{\prime}\right)^{\tau \wedge \tau^{\prime}} \mathbb{1}_{\left\{z_{0}=z_{0}^{\prime}\right\}}=\left(Z^{\prime}\right)^{\tau \wedge \tau^{\prime}} \mathbb{1}_{\left\{z_{0}=z_{0}^{\prime}\right\}},
$$

proving uniqueness of local mild solutions to (1.3).

Now, we suppose that $\left(\alpha, \sigma,\left.\gamma\right|_{B}\right)$ are locally Lipschitz and satisfy the linear growth condition. Let $z_{0}: \Omega \rightarrow H$ be an arbitrary $\mathcal{F}_{0}$-measurable random variable. By Lemma 4.3 , the mappings $\left(a, b,\left.c\right|_{B}\right)$ are also locally Lipschitz and satisfy the linear growth condition. Thus, by Theorem 3.14, there exists a strong solution $Y$ to (1.4) with initial condition $\ell z_{0}$. According to Proposition 4.4, the process $Z:=\pi U Y$ is a mild solution to (1.3) with initial condition $z_{0}$, proving the existence of mild solutions to (1.3).

If $\left(\alpha, \sigma,\left.\gamma\right|_{B}\right)$ are locally Lipschitz and locally bounded, then a similar proof, which uses Theorem 3.15, shows that existence of local mild solutions to (1.3) holds.

Remark 4.6. The structure $Z=\pi U Y$ shows that mild solutions to (1.3) obtained from Theorem 4.5 have càdlàg sample paths.

Remark 4.7. As pointed out in [5], the existence of weak solutions to (1.3) relies on a suitable stochastic Fubini theorem. Sufficient conditions can be found in [6].

\section{Acknowledgment}

The author is grateful to an anonymous referee for valuable comments and suggestions.

\section{References}

[1] G. Da Prato and J. Zabczyk, Stochastic Equations in Infinite Dimensions, vol. 44 of Encyclopedia of Mathematics and Its Applications, Cambridge University Press, Cambridge, Mass, USA, 1992.

[2] C. Prévôt and M. Röckner, A Concise Course on Stochastic Partial Differential Equations, vol. 1905 of Lecture Notes in Mathematics, Springer, Berlin, Germany, 2007. 
[3] X. Zhang, "Stochastic Volterra equations in Banach spaces and stochastic partial differential equation," Journal of Functional Analysis, vol. 258, no. 4, pp. 1361-1425, 2010.

[4] L. Gawarecki and V. Mandrekar, Stochastic Differential Equations in Infinite Dimensions with Applications to Stochastic Partial Differential Equations, Probability and Its Applications, Springer, Berlin, Germany, 2011.

[5] C. Marinelli, C. Prévôt, and M. Röckner, "Regular dependence on initial data for stochastic evolution equations with multiplicative Poisson noise," Journal of Functional Analysis, vol. 258, no. 2, pp. 616$649,2010$.

[6] D. Filipović, S. Tappe, and J. Teichmann, "Jump-diffusions in Hilbert spaces: existence, stability and numerics," Stochastics, vol. 82, no. 5, pp. 475-520, 2010.

[7] P. Kotelenez, "A submartingale type inequality with applications to stochastic evolution equations," Stochastics, vol. 8, no. 2, pp. 139-151, 1982/83.

[8] P. Kotelenez, "A stopped Doob inequality for stochastic convolution integrals and stochastic evolution equations," Stochastic Analysis and Applications, vol. 2, no. 3, pp. 245-265, 1984.

[9] C. Knoche, "SPDEs in infinite dimensional with Poisson noise," Comptes Rendus Mathématique. Académie des Sciences. Paris, vol. 339, no. 9, pp. 647-652, 2004.

[10] S. Peszat and J. Zabczyk, Stochastic Partial Differential Equations with Léry Noise, vol. 113 of Encyclopedia of Mathematics and Its Applications, Cambridge University Press, Cambridge, Mass, USA, 2007.

[11] S. Albeverio, V. Mandrekar, and B. Rüdiger, "Existence of mild solutions for stochastic differential equations and semilinear equations with non-Gaussian Lévy noise," Stochastic Processes and Their Applications, vol. 119, no. 3, pp. 835-863, 2009.

[12] C. I. Prévôt, "Existence, uniqueness and regularity w.r.t. the initial condition of mild solutions of SPDEs driven by Poisson noise," Infinite Dimensional Analysis, Quantum Probability and Related Topics, vol. 13, no. 1, pp. 133-163, 2010.

[13] J. Jacod and A. N. Shiryaev, Limit Theorems for Stochastic Processes, vol. 288 of Grundlehren der Mathematischen Wissenschaften, Springer, Berlin, Germany, 2nd edition, 2003.

[14] G. Cao, K. He, and X. Zhang, "Successive approximations of infinite dimensional SDEs with jump," Stochastics and Dynamics, vol. 5, no. 4, pp. 609-619, 2005.

[15] D. Applebaum, Lévy Processes and Stochastic Calculus, Cambridge University Press, Cambridge, Mass, USA, 2005.

[16] C. Dellacherie and P. A. Meyer, Probabilités et Potentiel, Hermann, Paris, France, 1982.

[17] R. K. Getoor, "On the construction of kernels," in Séminaire de Probabilités, IX, Lecture Notes in Mathematics 465, pp. 443-463, Springer, Berlin, Germany, 1975.

[18] D. Filipović, S. Tappe, and J. Teichmann, "Term structure models driven by Wiener processes and Poisson measures: existence and positivity," SIAM Journal on Financial Mathematics, vol. 1, pp. 523 $554,2010$.

[19] D. Filipovic, S. Tappe, and J. Teichmann, "Invariant manifolds with boundary for jump diffusions," http:/ / arxiv.org/abs/1202.1076.

[20] I. Karatzas and S. E. Shreve, Brownian Motion and Stochastic Calculus, vol. 113 of Graduate Texts in Mathematics, Springer, New York, NY, USA, 2nd edition, 1991.

[21] V. Mandrekar and B. Rüdiger, "Existence and uniqueness of path wise solutions for stochastic integral equations driven by Lévy noise on separable Banach spaces," Stochastics, vol. 78, no. 4, pp. 189-212, 2006.

[22] T. Yamada, "On the successive approximation of solutions of stochastic differential equations," Journal of Mathematics of Kyoto University, vol. 21, no. 3, pp. 501-515, 1981.

[23] T. Taniguchi, "Successive approximations to solutions of stochastic differential equations," Journal of Differential Equations, vol. 96, no. 1, pp. 152-169, 1992.

[24] B. Sz.-Nagy and C. Foiaş, Harmonic Analysis of Operators on Hilbert Space, North-Holland, Amsterdam, The Netherlands, 1970.

[25] E. B. Davies, Quantum Theory of Open Systems, Academic Press, London, UK, 1976.

[26] E. Hausenblas and J. Seidler, "Stochastic convolutions driven by martingales: maximal inequalities and exponential integrability," Stochastic Analysis and Applications, vol. 26, no. 1, pp. 98-119, 2008.

[27] E. Hausenblas and J. Seidler, "A note on maximal inequality for stochastic convolutions," Czechoslovak Mathematical Journal, vol. 51(126), no. 4, pp. 785-790, 2001. 


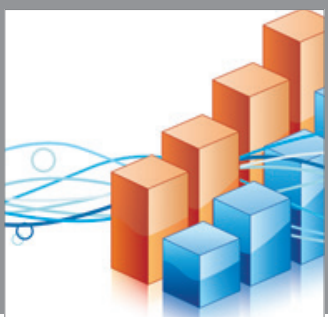

Advances in

Operations Research

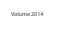

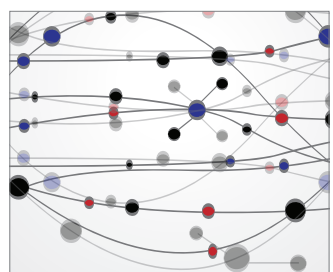

\section{The Scientific} World Journal
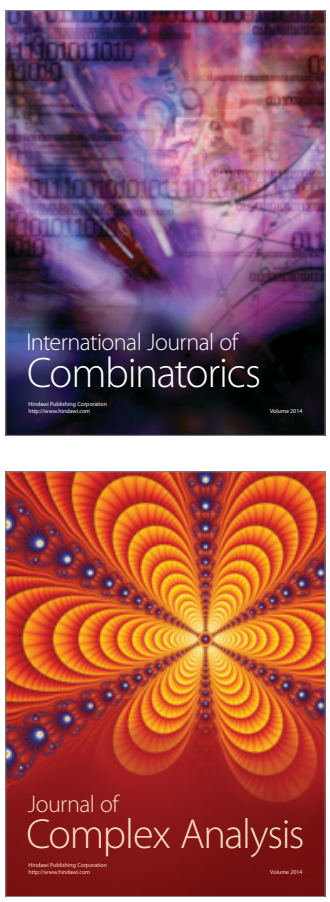

International Journal of

Mathematics and

Mathematical

Sciences
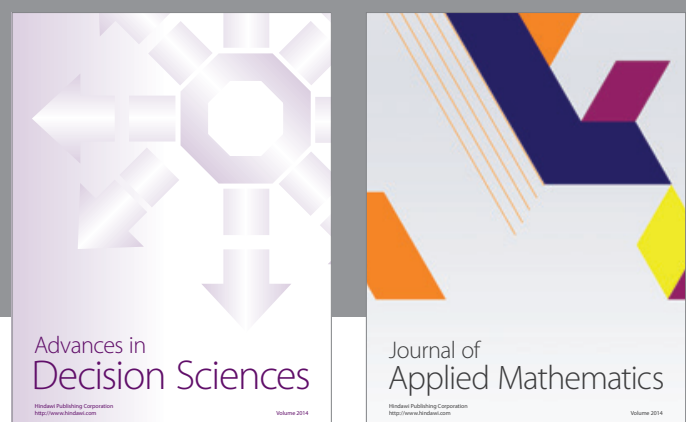

Journal of

Applied Mathematics
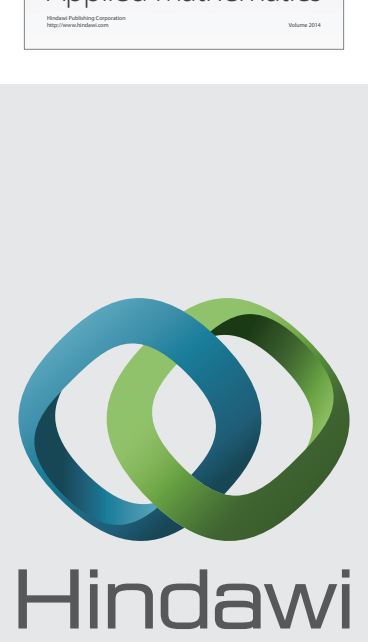

Submit your manuscripts at http://www.hindawi.com
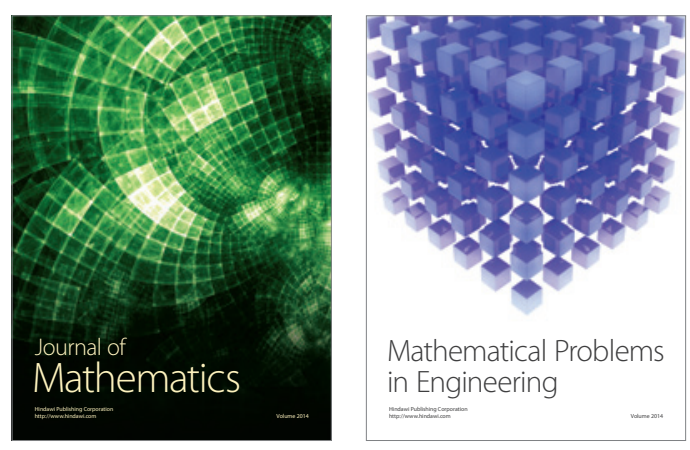

Mathematical Problems in Engineering
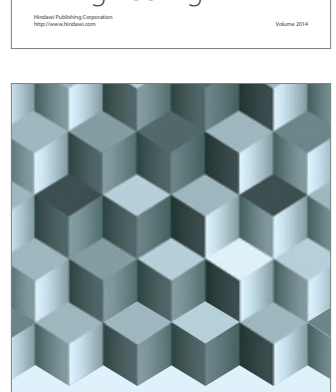

Journal of

Function Spaces
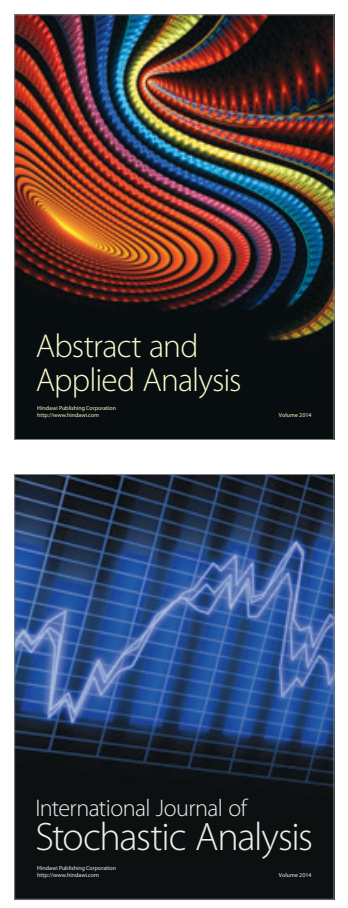

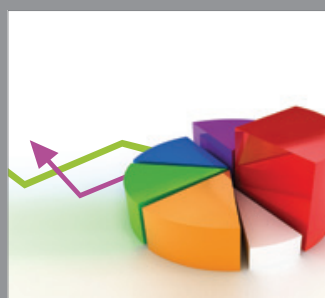

ournal of

Probability and Statistics

Promensencen
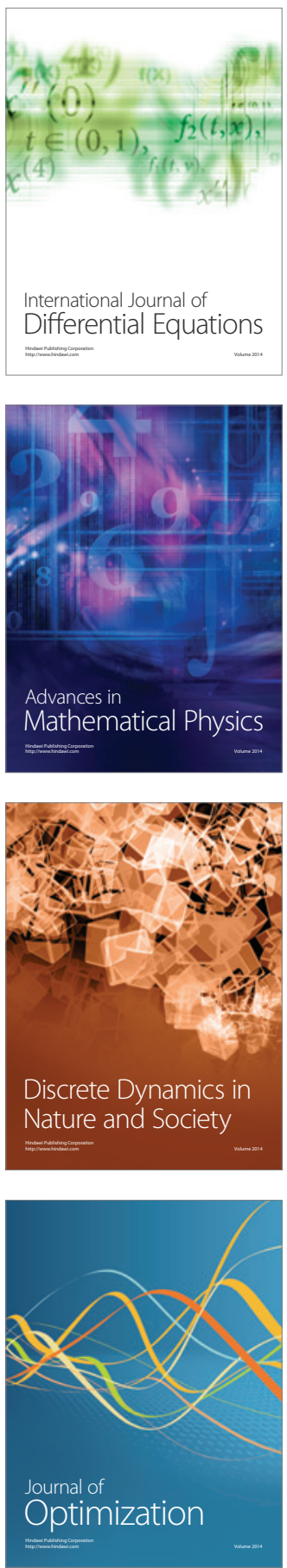